EPJ Web of Conferences 109,09001 (2016)

DOI: 10.1051/epjconf/201610909001

(C) Owned by the authors, published by EDP Sciences, 2016

\title{
Progress of Jinping Underground laboratory for Nuclear Astro- physics (JUNA)
}

WeiPing Liu ${ }^{1}$,a , ZhiHong $\mathrm{Li}^{1}$, JiangJun $\mathrm{He}^{2}$, XiaoDong Tang ${ }^{2}$, Gang $\mathrm{Lian}^{1}$, Zhu $\mathrm{An}^{4}$, JianJun Chang $^{2}$, Han Chen ${ }^{2}$, QingHao Chen ${ }^{3}$, XiongJun Chen ${ }^{1}$, ZhiJun Chen ${ }^{2}$, BaoQun Cui ${ }^{1}$, XianChao $\mathrm{Du}^{1}$, ChangBo Fu${ }^{5}$, Lin Gan ${ }^{1}$, Bing Guo ${ }^{1}$, GuoZhu He${ }^{1}$, Alexander Heger ${ }^{6}$, SuQing Hou ${ }^{2}$, HanXiong Huang ${ }^{1}$, Ning Huang ${ }^{4}$, BaoLu $\mathrm{Jia}^{2}$, LiYang Jiang ${ }^{1}$, Shigeru Kubono ${ }^{7}$, JianMin $\mathrm{Li}^{3}$, KuoAng $\mathrm{Li}^{2}$, Tao $\mathrm{Li}^{2}$, YunJu Li ${ }^{1}$, Maria Lugaro ${ }^{8}$, XiaoBing Luo ${ }^{4}$, Hong Yi Ma ${ }^{2}$, ShaoBo $\mathrm{Ma}^{2}$, DongMing $\mathrm{Mei}^{9}$, YongZhong Qian ${ }^{10}$, JiuChang Qin ${ }^{1}$, Jie Ren ${ }^{1}$, YangPing Shen ${ }^{1}$, Jun Su${ }^{1}$, LiangTing Sun ${ }^{2}$, WanPeng Tan ${ }^{11}$, Isao Tanihata ${ }^{12}$, Shuo Wang ${ }^{13}$, Peng Wang ${ }^{4}$, YouBao Wang ${ }^{1}$, Qi Wu ${ }^{2}$, ShiWei Xu ${ }^{2}$, ShengQuan Yan ${ }^{1}$, LiTao Yang ${ }^{3}$, Yao Yang ${ }^{2}$, XiangQing Yu ${ }^{2}$, Qian Yue ${ }^{3}$, Sheng Zeng ${ }^{1}$, HuanYu Zhang $^{1}$, Hui Zhang ${ }^{3}$, LiYong Zhang ${ }^{2}$, NingTao Zhang ${ }^{2}$, QiWei Zhang ${ }^{1}$, Tao Zhang ${ }^{5}$, XiaoPeng Zhang $^{5}$, XueZhen Zhang ${ }^{2}$, ZiMing Zhang ${ }^{2}$, Wei Zhao ${ }^{3}$, Zuo Zhao ${ }^{1}$, and Chao Zhou ${ }^{1}$

${ }^{1}$ China Institute of Atomic Energy, P. O. Box 275(1), Beijing 102413, China

${ }^{2}$ Institute of Modern Physics, Lanzhou, China

${ }^{3}$ Tsinghua University, Beijing, China

${ }^{4}$ Sichuan University, Chengdu, China

${ }^{5}$ Shanghai Jiaotong University, Shanghai, China

${ }^{6}$ Monash University, Melbourne, Victoria, Australia

${ }^{7}$ RIKEN, Institute of Physical and Chemical Research, Wako, Japan

${ }^{8}$ Konkoly Observatory of the Hungarian Academy of Sciences, Hungary

${ }^{9}$ South Dakota State University, Brookings, South Dakota, US

${ }^{10}$ Minnesota University, Minneapolis and Saint Paul, Minnesota, US

${ }^{11}$ University of Notre Dame, Notre Dame, Indiana, US

${ }^{12}$ Osaka University, Suita, Osaka, Japan

${ }^{13}$ Shandong University, Weihai campus, China

\begin{abstract}
Jinping Underground lab for Nuclear Astrophysics (JUNA) will take the advantage of the ultralow background in Jinping underground lab, high current accelerator based on an ECR source and highly sensitive detector to study directly a number of crucial reactions to the hydrostatic stellar evolution for the first time at their relevant stellar energies. In its first phase, JUNA aims at the direct measurements of ${ }^{25} \mathrm{Mg}(\mathrm{p}, \gamma)^{26} \mathrm{Al}$, ${ }^{19} \mathrm{~F}(\mathrm{p}, \alpha){ }^{16} \mathrm{O},{ }^{13} \mathrm{C}(\alpha, \mathrm{n}){ }^{16} \mathrm{O}$ and ${ }^{12} \mathrm{C}(\alpha, \gamma){ }^{16} \mathrm{O}$. The experimental setup, which include the accelerator system with high stability and high intensity, the detector system, and the shielding material with low background, will be established during the above research. The current progress of JUNA will be given.
\end{abstract}

a e-mail: wpliu@ ciae.ac.cn 


\section{Physical background}

Nuclear astrophysics is an interdisciplinary research field that integrates nuclear physics and astrophysics. The ultimate goal of nuclear astrophysics is to understand how nuclear processes generate the energy of stars over their lifetimes and, in doing so, synthesize heavier elements from the primordial hydrogen and helium in the big bang which led to the expanding universe [1]. Remarkable progress in understanding origin of elements and evolution of stars has been made since 1930's. However, there are still many scientific questions which need to be solved [2]. Direct measurement of the cross sections for the key nuclear reactions crucial to hydrostatic stellar evolution within Gamow window is important for obtaining benchmark data for stellar model, verifying extrapolation model, constraining theoretical calculations, and solving key scientific questions in nuclear astrophysics [3]. The direct measurement of astrophysical reaction rates on stable nuclei that require high-intensity beams and extremely low background, which represents enormous the major challenge at the frontiers of nuclear astrophysics. The largest challenge is the small cross section amid with large natural background. With the ultra-low background in deep underground environment, direct measurement of these key reactions in underground lab becomes a frontier in the field of experimental nuclear astrophysics. The first underground based low-energy accelerator facility, LUNA [4, 5] at Gran Sasso underground laboratory has successfully demonstrated the feasibility of meeting these challenges. Encouraged by the LUNA success, underground nuclear astrophysics has became one of the frontiers in the field of nuclear astrophysics. Relevant research programs are proposed in the long range plan in China, US and EU, with high priorities.

China JinPing underground Laboratory (CJPL) was established from a constructing hydro-power plants in the Jinping mountain, Sichuan, China [6,7]. The facility is located near the middle of traffic tunnel. The facility is shielded by $2400 \mathrm{~m}$ of mainly marble overburden, with radioactively quiet rock. Its ultra-low cosmic ray background, which is about 2 orders of magnitude lower than that in Gran Sasso, makes it into an ideal environment for low background experiment. CJPL phase I (CJPL-I) now housing CDEX [8] and PandaX dark matter experiments. CJPL phase II [9] (CJPL-II) is expected to be available by the beginning of 2016 for much larger scale underground experiments $\left(120,000 \mathrm{~m}^{3}\right.$ volume). JUNA will be one of its major research programs in CJPL-II.

CJPL provides a favorable condition to perform underground nuclear astrophysics experiment, thus JUNA was initiated. Currently, JUNA has been funded by National natural Science Foundation of China (NSFC), Chinese Academy of Sciences (CAS) and China National Nuclear (CNNC). JUNA team has long time experience of nuclear astrophysics, such as in-direct measurements based on Tandem accelerator, direct measurements based on $320 \mathrm{keV}$ platform, and accomplishing the first direct measurement of ${ }^{12} \mathrm{C}\left({ }^{12} \mathrm{C}, \mathrm{n}\right){ }^{23} \mathrm{Mg}$ at stellar energies.

\section{Scientific program}

The first phase of JUNA project aims at direct measurement of $(\alpha, \gamma)$ and $(\alpha, \mathrm{n})$, reactions in hydrostatic helium burning and $(\mathrm{p}, \gamma)$ and $(\mathrm{p}, \alpha)$ reactions in hydrostatic hydrogen and helium burning based on Jinping deep underground laboratory. Currently, many key reaction rates still suffer large uncertainties arising from the ambiguity of extrapolation. By combining the unparalleled depth and the ultra-low background environment of Jinping with the high current driven by an ECR source, we will establish a dedicated deep underground lab for the research of nuclear astrophysics.In the first phase, four key reactions, ${ }^{12} \mathrm{C}(\alpha, \gamma){ }^{16} \mathrm{O},{ }^{13} \mathrm{C}(\alpha, \mathrm{n}){ }^{16} \mathrm{O},{ }^{25} \mathrm{Mg}(\mathrm{p}, \gamma){ }^{26} \mathrm{Al}$ and ${ }^{19} \mathrm{~F}(\mathrm{p}, \alpha){ }^{16} \mathrm{O}$, will be studied for the first time within or near the astrophysical relevant energy regions (Gamow window). We expect to provide key inputs of nuclear physics for understanding evolution of stars and origin of element and solve some 
long standing problems in the field of nuclear astrophysics. In the following sections, the experimental plans are shown.

\section{$2.1{ }^{12} \mathbf{C}(\alpha, \gamma){ }^{16} \mathrm{O}$ reaction}

The ${ }^{12} \mathrm{C}(\alpha, \gamma){ }^{16} \mathrm{O}$ reaction is quoted as the holy grail in nuclear astrophysics [1]. The uncertainty of this reaction affects not only the nucleosynthesis of elements up to iron, but also the evolution of the massive stars and their final fate (black hole, neutron star). The cross section of this reaction has to be known within an uncertainty less than $10 \%$ at helium burning temperatures $\left(\mathrm{T}_{9}=0.2\right)$, corresponding to a Gamow window around $\mathrm{E}_{c . m .}=300 \mathrm{keV}$. It is extremely difficult to determine the reaction cross section (about $10^{-17}$ barn) at this energy [10]. Current technology can only achieve $10^{-14}$ barn cross section level. A direct measurement at $\mathrm{E}_{c . m .}=600 \mathrm{keV}$ near the Gamow window will be done in JUNA with high intensity ion beam of the experimental platform to provide better constrain for extrapolating models [11].

The main content of this research contains: 1) the measurement of angular distribution with high purity Germanium detectors (HPGe) at $\mathrm{E}_{c . m .}=600 \mathrm{keV}$ and the extrapolation to Gamow window via the R-matrix theory, 2) optimizing the experiment setup and the condition of environment (including the beam, the background shielding and the high-purity high power target) according to the results at $600 \mathrm{keV}$, measuring the total cross section at $\mathrm{E}_{c . m .}=600 \mathrm{keV}$ with $\mathrm{BGO}$ detection array to derive the cross section data with precision of $10 \%, 3$ ) test measurement at $\mathrm{E}_{c . m .}=380 \mathrm{keV}$ with $\mathrm{BGO}$ array.

For an angular distribution measurement at $\mathrm{E}_{c . m .}=600 \mathrm{keV}$, we plan to use ${ }^{4} \mathrm{He}^{2+}$ beam with an intensity of $5 \mathrm{emA}$ and an energy of $800 \mathrm{keV}\left(\mathrm{E}_{c . m .}=600 \mathrm{keV}\right)$ to bombard a high-purity ${ }^{12} \mathrm{C}$ target. Four or five HPGe detectors will be used to obtain the angular distribution of $\gamma$-rays emitted by ${ }^{12} \mathrm{C}(\alpha, \gamma){ }^{16} \mathrm{O}$ reaction. With this information, the R-matrix method will be applied to derive the contribution of the E1 and E2 components and extrapolate the cross section down to Gamow window. Fig. 1 shows the setup of four HPGe detectors and the high-purity ${ }^{12} \mathrm{C}$ target.

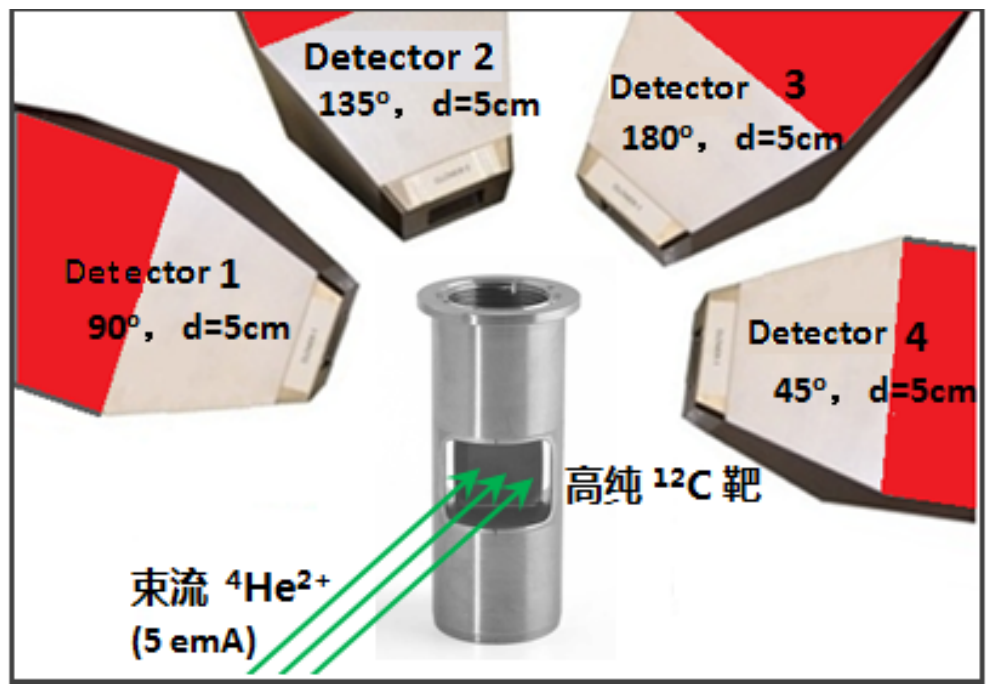

Figure 1. Schematic of ${ }^{12} \mathrm{C}(\alpha, \gamma){ }^{16} \mathrm{O}$ detection setup. 
For total cross section measurement at $\mathrm{E}_{c . m .}=600 \mathrm{keV}$, with the results of angular distribution measurement at $\mathrm{E}_{c . m .}=600 \mathrm{keV}$, we will optimize the experiment condition, including: 1) optimizing the beam transmission on the basis of the beam-optics calculation, adjusting the setup of shields to suppress the background coming from the beam, 2) confirming the origin of ${ }^{13} \mathrm{C}$ and improving the implantation condition of ${ }^{12} \mathrm{C}$ implantation target to reduce the disturbance of ${ }^{13} \mathrm{C}$. The BGO detection array placed around the target chamber can significantly increase the detection efficiency (absolute efficiency $75 \%$ at $\mathrm{E}_{\gamma}=6 \mathrm{MeV}$ ) of $\gamma$-rays. With the improvement above, an accurate total cross section will be obtained.

For total cross section test measurement at $\mathrm{E}_{c . m .}=380 \mathrm{keV}$, we will use ${ }^{4} \mathrm{He}^{2+}$ beam with an intensity of $5 \mathrm{emA}$ and an energy of $507 \mathrm{keV}\left(\mathrm{E}_{c . m .}=380 \mathrm{keV}\right)$ and the high-efficiency BGO detection array. A direct measurement of the total cross section of ${ }^{12} \mathrm{C}(\alpha, \gamma){ }^{126} \mathrm{O}$ in the energy region of near Gamow window will be tested.

\section{$2.2{ }^{13} \mathbf{C}(\alpha, \mathbf{n})^{16} \mathrm{O}$ reaction}

The ${ }^{13} \mathrm{C}(\alpha, \mathrm{n}){ }^{16} \mathrm{O}$ reaction is the key neutron source reaction for the stellar s-process nucleosynthesis. Due to the existence of sub-threshold resonances, there is a rather large uncertainty (30\%) in this important reaction rate which limits our understanding to the nucleosynthesis of heavy elements. We will take the advantage of the ultra low background in Jinping underground lab, the first underground high current accelerator based on an ECR source and high sensitive neutron detector to study directly this important reaction for the first time at energies down to $\mathrm{E}_{c . m} . \sim 0.2 \mathrm{MeV}$, within its relevant stellar energy range [12].

We are designing a fast neutron detector consisting of $24{ }^{3} \mathrm{He}$ proportional counters and a liquid scintillator. The schematic setup of the detector is shown in Fig. 2. The scintillator has a cylindrical shape with a length of $0.4 \mathrm{~m}$ and a diameter of $0.4 \mathrm{~m}$. The $24{ }^{3} \mathrm{He}$ counters are distributed in the two circles with radii of $0.1 \mathrm{~m}$ and $0.15 \mathrm{~m}$, respectively.

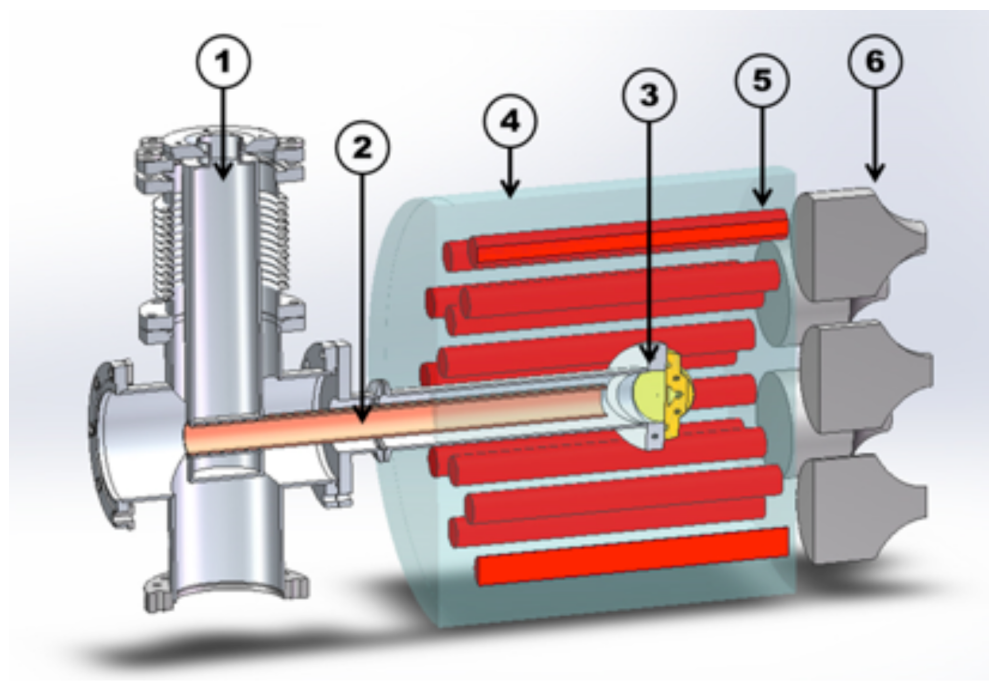

Figure 2. Schematic drawing of low background highly sensitive fast neutron detector. 1) $\mathrm{LN}_{2}$ cold trap; 2) Copper tube; 3) high power ${ }^{13} \mathrm{C}$ target; 4) Liquid scintillator; 5) ${ }^{3} \mathrm{He}$ detectors; 6) PMTs. 
The energies of neutrons from the ${ }^{13} \mathrm{C}(\alpha, \mathrm{n}){ }^{16} \mathrm{O}$ reaction are in the range of 2 to $3 \mathrm{MeV}$. The produced neutrons are firstly slowed down by the liquid scintillator. After their thermalization, some neutrons enter ${ }^{3} \mathrm{He}$ counters and are detected. With the coincidence between the fast signal from fast neutron slowing down inside the liquid scintillator and the delayed signal from the thermalized neutrons captured by the ${ }^{3} \mathrm{He}$ counters, we can effectively suppress the backgrounds in liquid scintillator and ${ }^{3} \mathrm{He}$ detectors. The detection efficiency after coincidence is estimated to be $20 \%$ for neutrons from the ${ }^{13} \mathrm{C}(\alpha, \mathrm{n}){ }^{16} \mathrm{O}$ reaction.

However, the coincidence cannot easily remove the correlated background. For example, in the decay chain of $\mathrm{U} / \mathrm{Th}$ impurities in the stainless steel walls of ${ }^{3} \mathrm{He}$ counters, there is a possibility that some product emits $\beta$ particle and then $\alpha$ decay. If the $\alpha$ particle enters the liquid scintillator while the $\beta$ triggers the liquid scintillator, a correlated event will be recorded. To suppress this kind of background, we plan to analyze the waveforms from ${ }^{3} \mathrm{He}$ counters to select the neutron events and reject $\alpha$ events. As a tradeoff, the efficiency of the ${ }^{3} \mathrm{He}$ counters will be decreased by a factor of 2. Therefore, the detection efficiency with coincidence between the ${ }^{3} \mathrm{He}$ counters and the liquid scintillator drops to $10 \%$.

\section{$2.3{ }^{25} \mathrm{Mg}(\mathrm{p}, \gamma){ }^{26} \mathrm{Al}$ reaction}

The ${ }^{25} \mathrm{Mg}(p, \gamma)^{26} \mathrm{Al}$ reaction is the main way to produce ${ }^{26} \mathrm{Al}$ in the galaxy and its cross section are dominated by the capture process of the isolated resonances in ${ }^{26} \mathrm{Al}$. The temperature range of astrophysical interests is $\mathrm{T}=0.02-2 \mathrm{GK}$, so the levels between $50 \mathrm{keV}$ and $310 \mathrm{keV}$ are more important in the study of galactic ${ }^{26} \mathrm{Al}$. Many experiments have been performed to study the ${ }^{25} \mathrm{Mg}(p, \gamma){ }^{26} \mathrm{Al}$ reaction sine 1970 [13-24], but the experiment on the surface of earth ground can only reach to 190 $\mathrm{keV}$ energy level due to the small cross section and large background effects of the cosmic rays. In 2012, the laboratory of underground nuclear astrophysics (LUNA) in Italy successfully measured the resonance strength at $92 \mathrm{keV}$ with the help of high shielding conditions in the underground laboratory $[25,26]$. However, the ${ }^{25} \mathrm{Mg}(p, \gamma)^{26} \mathrm{Al}$ cross section of $58 \mathrm{keV}$ resonant capture is inaccessible for direct measurement in the shielding conditions of LUNA experiments. The underground laboratory of Jinping in China covered with the marble rock of 2400 meters. Benefiting from the ultra low background and the high beam intensity, we will be able to measure the $58 \mathrm{keV}$ resonance strength of ${ }^{25} \mathrm{Mg}(p, \gamma){ }^{26} \mathrm{Al}$ with the new designed $4 \pi$ BGO $\gamma$ detectors array, as showing in Fig 3.

In order to optimize the experimental setup for the ${ }^{25} \mathrm{Mg}(p, \gamma)^{26} \mathrm{Al}$ reaction at the laboratory of Jinping underground nuclear astrophysics (JUNA), the resonance strength of $58 \mathrm{keV}$ level is estimated by using the shell model calculation. The results show that the $58 \mathrm{keV}$ resonance dominate the ${ }^{25} \mathrm{Mg}(p, \gamma)^{26} \mathrm{Al}$ reaction rate at $T<0.06 \mathrm{GK}$ [27]. The thick-target yield of the $58 \mathrm{keV}$ resonance of ${ }^{25} \mathrm{Mg}(p, \gamma)^{26} \mathrm{Al}$ with the calculated resonance strength is estimated under the conditions of JUNA with $10 \mathrm{~mA}$ proton beams and $4 \pi$ BGO $\gamma$-ray detector. The maximum yield is proportional to the resonance strength by

$$
Y_{\max }(\infty)=\frac{\lambda_{r}^{2}}{2} \omega \gamma \frac{M+m}{M \epsilon_{r}}
$$

where $\lambda_{r}$ and $\epsilon_{r}$ are the de Broglie wavelength and stopping power at resonant energy, $M$ and $m$ are the mass of the target nucleus and projectile, respectively. According to the calculation with Eq. (1), the effect statistical counting rate of the ${ }^{25} \mathrm{Mg}(p, \gamma)^{26} \mathrm{Al}$ reaction at $E_{\mathrm{c} . \mathrm{m} .}=58 \mathrm{keV}$ is about 1.4 events per day. The background counting rate is estimated to be less than 0.2 events per day. Thus we can accumulate around 40 reaction events in one month, and the statistic error will be about $16 \%$. 


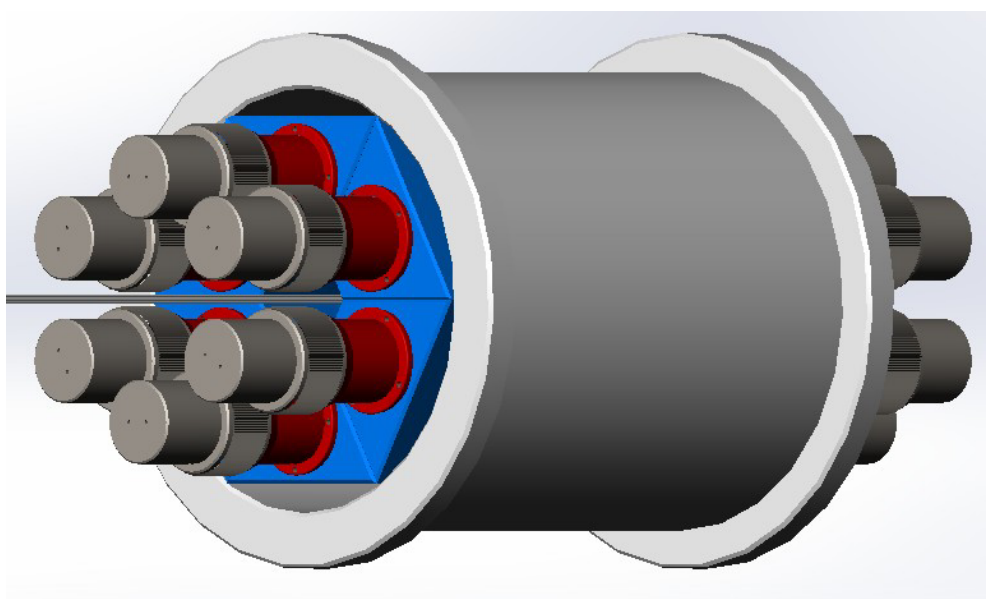

Figure 3. The $4 \pi$ BGO $\gamma$-ray detector designed for JUNA.

Table 1. Basic parameters of four reactions planned.

\begin{tabular}{l|cccccccc}
\hline reaction & beam & $\begin{array}{c}\text { intensity } \\
(\mathrm{emA})\end{array}$ & $\begin{array}{c}\text { c.m. energy } \\
(\mathrm{keV})\end{array}$ & cross section & target thickness & $\begin{array}{c}\text { efficiency } \\
\%\end{array}$ & $\begin{array}{c}\text { CTS } \\
\text { (/day) }\end{array}$ & $\begin{array}{c}\text { BKD } \\
\text { (/day) }\end{array}$ \\
\hline$\left.{ }^{12} \mathrm{C}(\alpha, \gamma)\right)^{16} \mathrm{O}$ & ${ }^{4} \mathrm{He}^{2+}$ & 2.5 & 380 & $10^{-13} \mathrm{mb}$ & $10^{18}$ atoms $/ \mathrm{cm}^{2}$ & 75 & 0.2 & 0.2 \\
${ }^{13} \mathrm{C}(\alpha, \mathrm{n})^{16} \mathrm{O}$ & ${ }^{4} \mathrm{He}^{1+}$ & 10 & 200 & $10^{-12} \mathrm{mb}$ & $10^{21}$ atoms $/ \mathrm{cm}^{2}$ & 20 & 7 & 1 \\
${ }^{25} \mathrm{Mg}(\mathrm{p}, \gamma)^{26} \mathrm{Al}$ & ${ }^{1} \mathrm{H}^{1+}$ & 10 & 58 & $\omega \gamma 2.1 \times 10^{-13} \mathrm{eV}$ & $0.6 \mu \mathrm{g} / \mathrm{cm}^{2}$ & 38 & 1.4 & 0.2 \\
${ }^{19} \mathrm{~F}\left(p, \alpha_{\gamma}\right){ }^{16} \mathrm{O}$ & ${ }^{1} \mathrm{H}^{1+}$ & 0.1 & 100 & $7.2 \times 10^{-9} \mathrm{mb}$ & $4 \mu \mathrm{g} / \mathrm{cm}^{2}$ & 75 & 27 & 0.2 \\
\hline
\end{tabular}

Table 2. Comparison of the goal for four reaction with current status.

\begin{tabular}{l|cccccc}
\hline reaction & physics & $\begin{array}{c}\text { current } \\
\text { energy limit }(\mathrm{keV})\end{array}$ & $\begin{array}{c}\text { precision } \\
(\%)\end{array}$ & ref. & $\begin{array}{c}\text { JUNA } \\
\text { energy limit }(\mathrm{keV})\end{array}$ & $\begin{array}{c}\text { precision } \\
(\%)\end{array}$ \\
\hline${ }^{12} \mathrm{C}(\alpha, \gamma){ }^{16} \mathrm{O}$ & Massive star & 890 & 60 & {$[29]$} & 380 & test \\
${ }^{13} \mathrm{C}(\alpha, \mathrm{n})^{16} \mathrm{O}$ & Heavy ion synthesis & 279 & 60 & {$[30]$} & 200 & 20 \\
${ }^{25} \mathrm{Mg}(\mathrm{p}, \gamma)^{26} \mathrm{Al}$ & Galaxy ${ }^{26} \mathrm{Al}$ source & 92 & 20 & {$[25]$} & 58 & 15 \\
${ }^{19} \mathrm{~F}\left(p, \alpha_{\gamma}\right)^{16} \mathrm{O}$ & F overabundance & 189 & 80 & {$[31]$} & 100 & 10 \\
\hline
\end{tabular}

\section{$2.4{ }^{19} \mathbf{F}(p, \alpha){ }^{16} \mathrm{O}$ reaction}

The ${ }^{19} \mathrm{~F}(p, \alpha){ }^{16} \mathrm{O}$ reaction is considered to be an important reaction in the CNO cycles. Currently, the experimental cross sections of this reaction at Gamow energies are still incomplete, and the precision of its thermonuclear reaction rate does not yet satisfy the model requirement. The proposed experiment is targeting on direct cross section measurement of the key ${ }^{19} \mathrm{~F}(p, \alpha){ }^{16} \mathrm{O}$ reaction right down to the Gamow energies (70-350 keV in the center-of-mass frame) with a precision better than $10 \%$ [28].

A 'lamp'-type Micron silicon array will be constructed for the charged particle measurement, which can cover about $4 \pi$ solid angle. This universal detection array will set the base for studying the charged-particle-induced reactions at JUNA. A conceptual design is shown in Fig. 4. It can not only measure the total $\left(p, \alpha_{0}\right)$ cross section but also the angular distribution. The experimental angular distribution is very useful for revealing nuclear structure of the low-energy resonances. In this experiment, a thin target of about $4 \mu \mathrm{g} / \mathrm{cm}^{2} \mathrm{CaF}_{2}$ will be utilized, which is evaporated on a thin metal 
backings. Thanks to the high $Q$ value (about $8.11 \mathrm{MeV}$ ) for this reaction, the average energy for the emitted $\alpha$ particles is about $6.7 \mathrm{MeV}$. These relatively high-energy particles can penetrate the backings and be detected easily at the forward angle. The detectors at the forward angle do not face the Rutherford-scattered strong proton beam which is stopped in the backings. However, those detectors at the backward angle should be shielded by a thin foil, e.g., a mylar foil, to stop the scattered protons. The target backing will be connected to a cooling device to release the heat during the experiment.

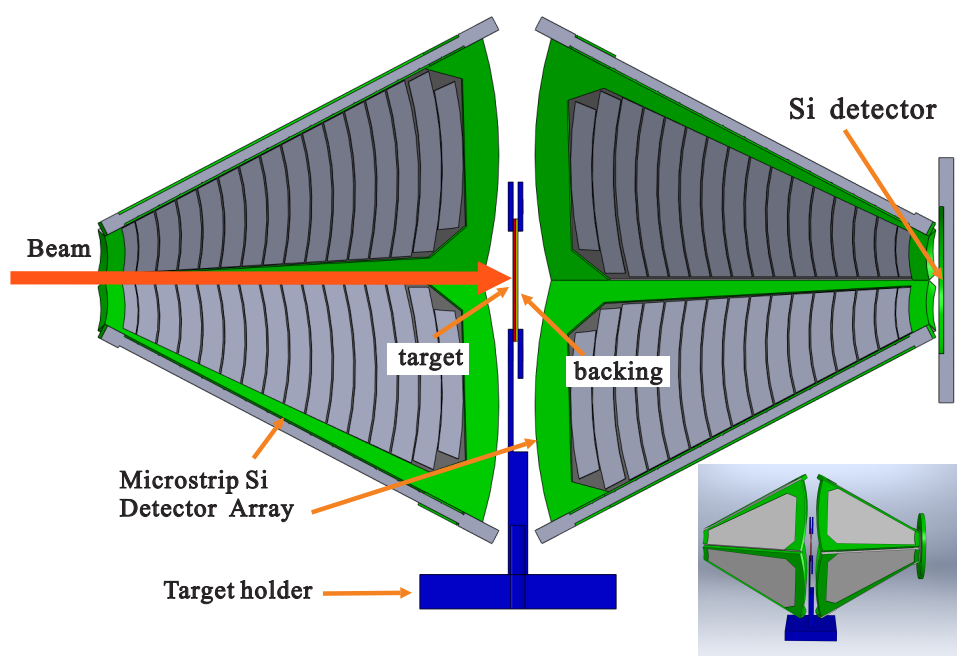

Figure 4. Conceptual silicon detector array designed for measuring the charged particles.

As for the ${ }^{19} \mathrm{~F}\left(p, \alpha_{\gamma}\right){ }^{16} \mathrm{O}$ channel, the energies of emitted $\gamma$ rays are about 6-7 MeV. In this project, two $\gamma$ detection arrays will be constructed: one is a HPGe array whose absolute detection efficiency is about $1 \%$ for the $\gamma$ rays of interest with excellent energy resolution; another one is the $4 \pi$ BGO array as introduced above, whose absolute efficiency is about $75 \%$, but with moderate resolution. Here, the HPGe array will be utilized in the $E_{c . m .}>140 \mathrm{keV}$ energy region, while the BGO array will be used below this energy region. With the excellent resolution of the HPGe detector, the possible contaminations can be resolved and identified clearly, which makes the BGO $\gamma$-ray identification reliable at lower-energy region. A conceptual design for the HPGe array is shown in Fig. 5.

The preliminary studies will by done based on the $320 \mathrm{kV}$ platform at IMP Lanzhou. In the period of 2015-2016, several tests for the proposed experiment will be carried out, in order to check: (1) the stability of the thin $\mathrm{CaF}_{2}$ target against the high current, about several tens of $\mu \mathrm{A}$ of proton; (2) the contaminants in the forward angle; (3) the chemical compositions of the target. As scheduled, we will make a campaign for the experimental measurement in 2017, and publish the results in 2019.

\subsection{Summary of the four reactions}

The counting rate and background of four reactions are estimated. The counting rate are estimated according to the updated evaluation and/or the best extrapolation. The counting rate are deduced from the CJPL-I environment and detector $\gamma$ and neutron measurement data. The results are summarized 


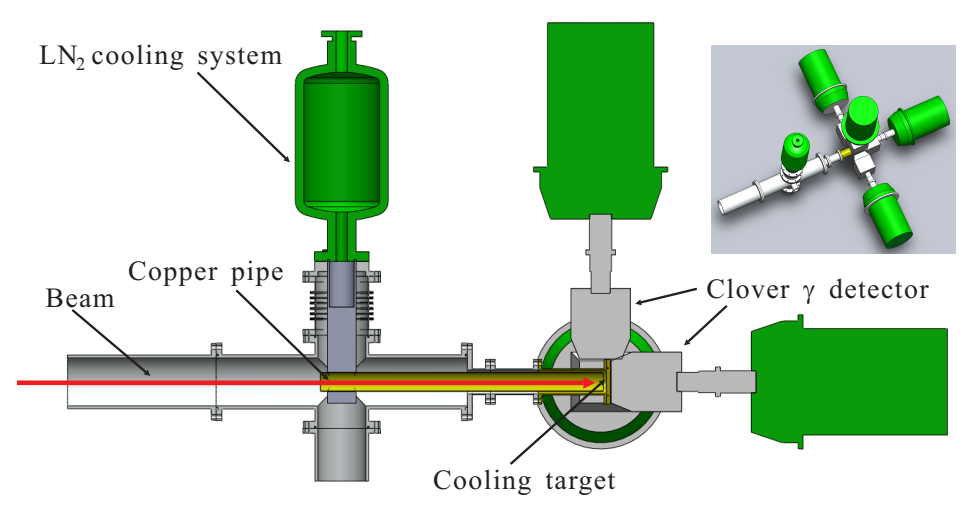

Figure 5. Conceptual HPGe detector array designed for measuring the $\gamma$ rays.

in Tab. 1. We also compared our expected precision of data with current experimental results. The results are summarized in Tab. 2.

\section{Accelerator, detector and shielding system}

The key techniques, which including the accelerator system with high stability and high intensity, the detector detection system, and the shielding material with ultra-low background, will be developed through the above research.

The preliminary design of the low energy and high current accelerator system of JUNA is shown in Fig. 6. For ensuring the four reaction measurement, we plan to optimize the accelerator system based on the following consideration [32].

\subsection{GHz ECR ion source and Low Energy Beam Trasport Line}

We adopted a design of $2.45 \mathrm{GHz}$ ECR which is developed to CI-ADS project. This ion source is expected to delivered $12 \mathrm{emA}$ proton, $6 \mathrm{emA} \mathrm{He}^{+}$and $2.5 \mathrm{emA} \mathrm{He}^{2+}$. The maximum beam energy out of ion source is $50 \mathrm{keV} / \mathrm{q}$ with emittance less than $0.2 \pi \cdot \mathrm{mm} \cdot \mathrm{mrad}$. The Low Energy Beam Transport line (LEBT) is designed to minimize the space charge effect and improve the beam transport efficiency. Beam will be accelerated before being focused with two solenoids. To keep the LEBT as short as possible, all the steering magnets are built inside of the solenoids. $\mathrm{He}^{2+}$ beam is expected to be mixed with a large fraction of $\mathrm{He}^{+}$beam. A $30 \mathrm{deg}$ magnet will be added between the two solenoids to filter out the intense $\mathrm{He}^{+}$to reduce the burden of the acceleration tube.

\subsubsection{Developing a high stability power supply system}

For the nuclear reaction measurement planed near Gamow window, the long-term stability is the key to achieve the high measurement precision. Hence we must use the high-voltage power supply with 


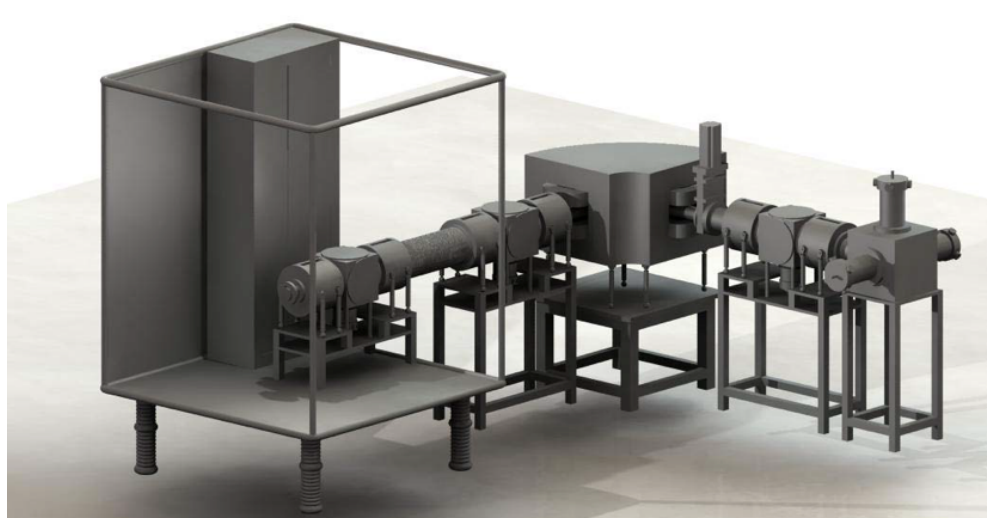

Figure 6. Design of the low energy and high current accelerator system.

high stability. We plan to cooperate with Glassman High Voltage, Inc and develop a $400 \mathrm{kV}, 6 \mathrm{~kW}$ high stability power supply (long-term output voltage stability $0.05 \%$, ripple voltage $0.01 \%$ ).

\subsubsection{Optimizing the design of the accelerating tube}

As for the low energy and high intensity beam, the space-charge effect must be controlled during transmission in order to increase the transport efficiency. The high transport efficiency could not only ensure enough beam intensity on target, but also reduce the background brought by the beam itself. We plan to adopt segmental voltage for the accelerating tube and design an acceleration and deceleration structure for the accelerating tube electrode to reduce the space-charge effect.

\subsubsection{The design and installation of the experimental shielding system}

The effect to background ratio of the nuclear reaction measurement will be significantly enhanced with the ultra-low background of CJPL and high current beam. But at the same time the high current beam will bring new background, which must be shielded. We plan to construct two shielding system around the target chamber and the detectors, aiming at shielding $\gamma$-ray and neutron, respectively.

In order to avoid the influence to other laboratories in CJPL, we plan to cover the accelerating tube with Lead shielding layer and build a concrete shielding wall in our laboratory to insulate the background coming from accelerator.

\subsubsection{The development of the high power solid target}

In order to keep the stability of the solid target under the bombardment of high current mA level beam, we plan to develop a high power solid target system. The temperature of target will be effectively controlled by careful design of heat conduction and water cooling device. The design power of the superpower solid target system is $20 \mathrm{~kW} / \mathrm{cm}^{2}$, which can satisfy the requirement of the four experiments in the JUNA project. 


\section{Summary and conclusion}

The accelerator system and detector array will be installed in 2017, experiment will be started in 2018 and the first batch of experimental results will be delivered in 2019.

In summary, a new underground nuclear astrophysics experiment JUNA planned for the expanded space CJPL-II. It is planned to set up a particle accelerator and detectors used to replicate the nuclear processes generating energy within stars and the synthesis of heavier elements from hydrogen and helium in the primordial universe. The rock shielding would reduce background noise, making it easier for researchers to detect rare and subtle signals. With a more powerful accelerator and a deeper location than other efforts, JUNA has the potential to take a favorable position among underground nuclear astrophysics labs.

\section{References}

[1] Rolfs C and Rodney W S, Cauldrons in the Cosmos, The University Chicago Press, 1988

[2] Haseltine E, Discover Magazine, 2002, 23: 2

[3] Iliadis C, Nuclear Physics of Stars, Wiley-VCH Verlag GmbH, 2007

[4] Formicola A, Nuclear Instruments \& Methods in Physics Research, 2003, 507(3):609-616.

[5] Costantini H, Formicola A, Imbriani G, et al. Reports on Progress in Physics, 2009, 972(8):086301.

[6] Chen H S, Science(in Chinese), 2010, 62: 4

[7] Cheng J P, Wu S Y, Yue Q, et al. Physics (in Chinese), 2011, 03(03):149-154.

[8] Zhao W, Yue Q, Kang K J, et al. Physical Review D, 2013, 88(5):1201-1205.

[9] Normile D. Science, 2014, 346(6213):1041-1041.

[10] Buchmann L, et al., Extrapolation, NIC proceedings

[11] Liu W P, The ${ }^{12} \mathrm{C}(\alpha, \gamma){ }^{16} \mathrm{O}$ reaction proporsal, 2014, unpublished.

[12] Tang X D, The ${ }^{13} \mathrm{C}(\alpha, \mathrm{n}){ }^{16} \mathrm{O}$ reaction proporsal, 2014, unpublished.

[13] Betts R R, Fortune H T, Pullen D J. Nuclear Physics A, 1978, 299(3):412-428.

[14] Champagne A E, Howard A J, Parker P D. Nuclear Physics A, 1983, 402(1):159178.

[15] Champagne A E, Howard A J, Parker P D. Nuclear Physics A, 1983, 402(1): 179188.

[16] Endt P M, de Wit P, Alderliesten C. Nuclear Physics A, 1986, 459(1):61-76.

[17] Champagne A E, Nald A B, Wang T F, et al. Nuclear Physics A, 1986, 451(3):498-508.

[18] Endt P M, Rolfs C. Nuclear Physics A, 1987, 467(2):261272.

[19] Champagne A E, Howard A J, Smith M S, et al. Nuclear Physics A, 1989, 505(2):384396.

[20] Rollefson A A, Wijekumar V, Browne C P, et al. Nuclear Physics A, 1990, 507(2):413425.

[21] Iliadis C, Schange T, Rolfs C, et al. Nuclear Physics A, 1990, 512(3):509530.

[22] Iliadis C, Buchmann L, Endt P M, et al. Physical Review C, 1996, 53(1):475-496.

[23] Powell D C, Iliadis C, Champagne A E, et al. Nuclear Physics A, 1998, 644(4):263276.

[24] Arazi A, Faestermann T, Niello J O F, et al. Physical Review C, 2006, 74(2):025802.

[25] Strieder F, Limata B, Formicola A, et al. Physics Letters B, 2012, 707:6065.

[26] Straniero O, Imbriani G, Strieder F, et al. Astrophysical Journal, 2013, 763(2).

[27] Li Z H, Su J, Li Y J, et al. Science China Physics, Mechanics \& Astronomy, 2015, 58.

[28] He J J, The ${ }^{19} \mathrm{~F}(\mathrm{p}, \gamma){ }^{16} \mathrm{O}$ reaction proporsal, 2014, unpublished.

[29] Hammer J W, Fey M, Kunz R, et al. Nuclear Physics A, 2005, 758:363366.

[30] Drotleff H W, Denker A, Knee H, et al. Astrophysical Journal, 1993, 414:735-739. 


\section{OMEG2015}

[31] Lombardo I, Dell'Aquila D, Leva A D, et al. Physics Letters B, 2015:178182.

[32] Lian G, The JUNA platform proporsal, 2014, unpublished. 